\title{
Diagnostic imaging findings of pelvic retroperitoneal ganglioneuroma in a child: a case report with the emphasis on initial ultrasound findings
}

\author{
Deniz Turkyılmaz Mut ${ }^{1}$, Umut Percem Orhan Soylemez ${ }^{2}$, Mesut Demir ${ }^{3}$, Canan Tanık ${ }^{4}$, \\ Alper Ozel ${ }^{1}$
}

${ }^{1}$ Sisli Hamidiye Etfal Training and Research Hospital, Department of Radiology, Istanbul, ${ }^{2}$ Bingol State Hospital, Department of Radiology, Bingol, ${ }^{3}$ Sisli Hamidiye Etfal Training and Research Hospital, Department of Pediatric Surgery, Istanbul, ${ }^{4}$ Sisli Hamidiye Etfal Training and Research Hospital, Department of Pathology, Istanbul, Turkey

\footnotetext{
Abstract

Ganglioneuromas are rare benign tumors of neural crest origin developed along the sympathetic chain. The pelvic retroperitoneum is the rarest location of these tumors. Clinically these tumors are commonly asypmtomatic even if they reach large sizes. Here we report the radiological features of a 16 year old boy with pathologically proven retroperitoneal ganglioneuroma that was detected initially by ultrasound. Relevant literature is also discussed.

Keywords: ganglioneuroma, pelvic, ultrasonography, CT, MRI
}

\section{Introduction}

Ganglioneuromas are rare benign tumors of neural crest origin. They belong to a group of neoplasm that exhibit a wide range of differentiation with ganglioneuroma being benign and neuroblastoma, malignant. They develop along the sympathetic chains and their usual locations are mediastinum, neck, retroperitoneum, and rarely the pelvis. Ganglioneuromas are commonly asymptomatic, and the development of clinical symptoms depend on the localisation and the size of the tumour. Additionally, because of the malignant transformation of a small number of ganglioneuromas reported in the literature, early diagnosis and complete excision of the tumour is essentially important [1-4].

We report the case of a 16 year old boy admitted with bilateral loin pain due to a pelvic ganglioneuroma detected

Received 12.05.2015 Accepted 03.06.2015

Med Ultrason

2016, Vol. 18, No 1, 120-122

Corresponding author: Alper Ozel

Halaskargazi Cad, Etfal Sokak, Sisli Hamidiye Etfal Egitim ve Arastirma Hastanesi

34377 Sisli / Istanbul, Turkey

Phone: 00902123735000 / 4624

E-mail: dralperozel@gmail.com initially at abdominopelvic ultrasonography. The additional imaging findings on computed tomography (CT) and magnetic resonance imaging (MRI) of this rare tumour were revealed and discussed in view of the literature.

\section{Case report}

A 16 year old male complaining of bilateral flank pain was referred to ultrasonography department. Physical examination and laboratory tests revealed no abnormality. Abdominal ultrasound was requested to explain the flank pain. Abdominopelvic ultrasonography revealed a $8 \times 5 \times 5 \mathrm{~cm}$ sized well defined round to ovoid hypoechoic solid mass in the right half of pelvis adjacent to the common iliac vasculature (fig 1a,b). Doppler ultrasonography showed mild vascularity within the lesion and low resistance arterial flow (fig 1c). Contrast enhanced CT and MR imaging were performed to further clarify the nature of the lesion. CT revealed a well defined mass located in the right low quadrant, medial of the common iliac arteries. Slightly extension to paravertebral space was observed. The tumor was homogeneous with relatively low attenuation and did not enhance by intravenous contrast medium (fig 1d). MRI revealed that the lesion showed homogeneous low signal intensity on T1-weighted images and inhomogeneous high 


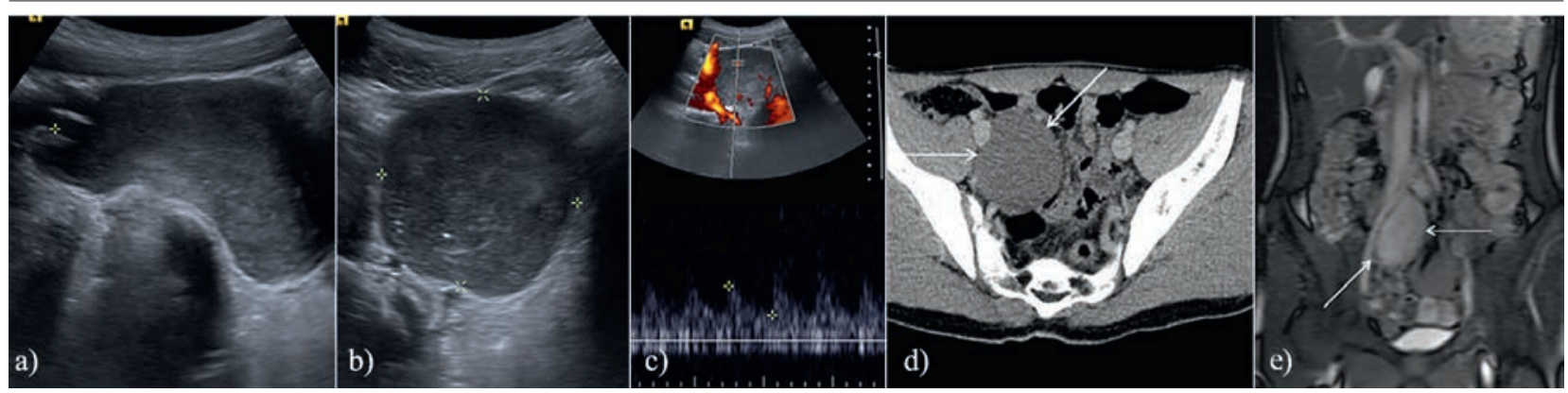

Fig 1. a) Sagittal abdominal ultrasonography revealed a $8 \times 5 \times 5 \mathrm{~cm}$ sized well defined round to ovoid hypoechoic solid mass in the right lower quadrant adjacent to common iliac vasculature; b) Transverse abdominal ultrasonography; c) Doppler ultrasonography showed mild vascularity and low resistance arterial flow d) CT revealed a well defined hypodense mass lesion (white arrows) located medially to the right common iliac vessels. The tumor was homogeneous with relatively low attenuation without enhancement after intravenous contrast medium administration; e) coronal T2WI revealed a hyperintense lesion, which enhanced mildly on the delayed postcontrast T1WI.

signal intensity on T2-weighted images and showed mild delayed enhancement (fig 1e). There was no suggestion of lymph node involvement or ascites. Retroperitoneal neurogenic tumor was assumed as the diagnosis and the patient underwent surgery. With an anterior transperitoneal approach, the lesion was reached in the pelvic retroperitoneum. A 8x5x5 cm sized well contoured, round, fixed mass was observed. It was removed completely from adjacent structures with blunt excision. Histopathology revealed a mature ganglion cell in a schwannian rich stromal background, indicating mature ganglioneuroma.

\section{Discussions}

Ganglioneuromas are benign slow growing tumours that arise from sympathetic ganglion cells. They are considered to be part of neuroblastoma group together with neuroblastomas and ganglioneuroblastomas [5]. They develop along the sympathetic chain, and the most common locations in decreasing order are abdomen (52\%)-including adrenal and extra-adrenal sites-, mediastinum (39\%) and rarely pelvis or neck (9\%) [6].

Clinically, ganglioneuromas are usually asymptomatic even if they reach a large size [7,8]. Sometimes, if retroperitoneal in location the abdominal pain, palpation of an abdominal mass or compression of neighbouring structures may suggest the diagnosis. Although ganglioneuromas are mostly nonfunctioning, some show metabolic activity caused by vasoactive peptides and present with such symptoms such as hypertension or diarrhea. The reported symptoms in pelvic ganglioneuromas especially in presacral location are pain, constipation or amenorrhea, but are mostly clinically silent [9]. In our patient, pelvic ganglioneuroma was incidentally detected on abdominopelvic ultrasonography performed to clarify bilateral loin pain.
Ultrasound is the initial diagnostic modality in screening and detecting retroperitoneal and pelvic ganglioneuromas, especially in the pediatric population. Its lack of ionizing radiation, safeness, noninvasiveness, and ready availability are the major advantages over other modalities. However, the sonographic appearance is nonspecific as showing an heterogenous hypoechoic solid lesion. Ganglioneuromas are reported as hypovascular tumours [10]; however, there is lack of information about the Doppler ultrasonographic features of these lesions in the literature. In our case, Doppler ultrasonography displayed scarce arterial vascularity with low resistance waveform.

On CT and MR images, ganglioneuromas usually present as oval, crescentic or lobulated paravertebral masses, when located retroperitoneally. CT is very sensitive in the detection of calcifications. In $20 \%$ to $30 \%$ of ganglioneuromas calcifications are present, which are typically fine and speckled rather than amorphous and coarse as in neuroblastomas [11-13]. Intraspinal extension of ganglioneuromas are considered frequent as reported in some publications. In a series of ganglioneuromas by Cai et $\mathrm{al}$, some ganglioneuromas tended to extend to intervessel space without encasing them. This was interpreted as an important imaging manifestation reflecting the soft and benign biological behaviour of these lesions [1].

Additionally, the authors demonstrated low attenuation on unenhanced CT in all ganglioneuromas and correlated the measured CT Hounsfield value to pathologic examination. A large amount of myxoid stroma with relatively few cellular components and collagen fibers was found in tumors with $\mathrm{CT}$ value less than 30 Hounsfield unit [1].

The MR imaging characteristics of ganglioneuromas reported previously were hypointensity on T1WI and hyperintensity on T2WI. The histologic compositions of ganglioneuromas with an abundant amount of myxoid stroma and a relatively small amount of Schwann and 
ganglion cell components is postulated to be responsible for the signal characteristics on MRI $[2,14]$. On CT and MRI, the ganglioneuromas demonstrate delayed enhancement pattern to a varying degree. In the study of Cai et al, they claimed that the enhancement feature could be explained by the presence of an abundant amount of myxoid stroma in the ganglioneuromas, resulting in delayed progressive accumulation of contrast in the extracellular space. Contrary to ganglioneuromas, neuroblastomas and ganglioneuroblastomas demonstrate often more inhomogenous enhancement in the early phase after injection of contrast media [1]. Although the contrast enhancement pattern on MRI was in accordance with that reported in the literature, lack of enhancement on $\mathrm{CT}$ was thought to be the administration of a single phase $\mathrm{CT}$ protocol in pediatric patients in our institution.

The differential diagnosis list of retroperitoneal ganglioneuromas with pelvic extension include neuroblastoma, ganglioneuroblastoma, schwannoma, meningioma, or other cystic lesions [9]. FNA can be used preoperatively, but it usually leads to inconclusive diagnosis. In the largest series with ganglioneuromas of presacral location, the diagnosis could not be achieved in three patients with FNA [15]. Without intention to perform FNA due to inconclusive results, a benign neurogenic tumor was considered as the presumed diagnosis according to CT and MRI features and lack of enhancement of the lesion.

Surgical resection represent the choice for treatment. Because of the benign nature of ganglioneuromas, adjuvant systemic chemotherapy or local radiotherapy are not indicated. All pelvic ganglioneuromas above the third sacral vertebral body level should be excised by anterior abdominal approach. When ganglioneuromas are located below the middle of S3 vertebra body level, posterior sacral transection or combined anterior and posterior approach is the ideal treatment, unless with pelvic viscera involvement [16].

In conclusion, retroperitoneal pelvic ganglioneuroma is an uncommon benign pathology which is derived from neural crest cells. Especially in the pediatric age group, Doppler ultrasound is usually the initial modality used for detection of these hypovascular tumors, showing their origin and relationships to the neighbouring organs. The delayed enhancement pattern on CT and/or MRI is a helpful feature in reaching the correct diagnosis. Surgery is the primary means of diagnosis and treatment.

\section{References}

1. Cai J, Zeng Y, Zheng H, Qin Y, T K, Zhao J. Retroperitoneal ganglioneuroma in children: CT and MRI features with histologic correlation. Eur J Radiol 2010; 75: 315-320.

2. Otal P, Mezghani S, Hassissene S, et al. Imaging of retroperitoneal ganglioneuroma. Eur Radiol 2001; 11: 940-945.

3. Moschovi M, Arvanitis D, Hadjigeorgi C, Mikraki V, Tzortzatou-Stathopoulou F. Late malignant transformation of dormant ganglioneuroma? Med Pediatr Oncol 1997; 28: 377-381.

4. Kimura S, Kawaguchi S, Wada T, Nagoya S, Yamashita T, Kikuchi K. Rhabdomyosarcoma arising from a dormant dumbbell ganglioneuroma of the lumbar spine: a case report. Spine 2002; 27: E513-E517.

5. Lynch NP, Neary PM, Fitzgibbon JF, Andrews EJ. Successful management of presacral ganglioneuroma: A case report and a review of the literature. Int J Surg Case Rep 2013; 4: 933-935.

6. Yamaguchi K, Hara I, Takeda M, et al. Two cases of ganglioneuroma. Urology 2006; 67: 622.e1-4.

7. Schulman H, Laufer L, Barki Y, et al. Ganglioneuroma: an 'incidentaloma' of childhood. Eur Radiol 1998; 8: 582-584.

8. Chang CY, Hsieh YL, Hung GY, Pan CC, Hwang B. Ganglioneuroma presenting as an asymptomatic huge posterior mediastinal and retroperitoneal tumor. J Chin Med Assoc 2003; 66: 370-374.

9. Cerullo G, Marrelli D, Rampone B, et al. Presacral ganglioneuroma: a case report and review of literature. World $\mathrm{J}$ Gastroenterol 2007; 13: 2129-2131.

10. Balaj C, Oliver A, Lemarie C, Hubert J, Laurent V, Regent D. Retroperitoneal ganglioneuroma revealed as an incidentaloma in a healthy volunteer. Diagn Interv Imaging 2015; 96: 93-96.

11. Ichikawa T, Ohtomo K, Araki T, et al. Ganglioneuroma: CT and MR features. Br J Radiol 1996; 69: 114-121.

12. Radin R, David CL, Goldfarb H, Francis IR. Adrenal and extra-adrenal retroperitoneal ganglioneuroma: imaging findings in 13 adults. Radiology 1997; 202: 703-707.

13. Scherer A, Niehues T, Engelbrecht V, Modder U. Imaging diagnosis of retroperitoneal ganglioneuroma in childhood. Pediatr Radiol 2001; 31: 106-110.

14. Van Dyck P, Op de Beeck B, Parizel PM. Helical CT and dynamic MR features of an adrenal ganglioneuroma. JBRBTR 2006; 89: 77-79.

15. Modha A, Paty P, Bilsky MH. Presacral ganglioneuromas. Report of five cases and review of the literature. J Neurosurg Spine 2005; 2: 366-371.

16. Vardas K, Manganas D, Papadimitriou G, et al. Presacral ganglioneuroma: diagnostic considerations and therapeutic strategy. Case Rep Oncol 2013; 6: 561-568. 